\title{
Aluminum nitride hydrolysis enabled by hydroxyl-mediated surface proton hopping
}

Christopher J. Bartel ${ }^{1}$, Christopher L. Muhich ${ }^{1,2}$, Alan W. Weimer ${ }^{1 *}$, and Charles B. Musgrave $\mathrm{e}^{1,3^{*}}$

${ }^{I}$ Department of Chemical and Biological Engineering, University of Colorado, Boulder, Colorado 80309, United States

${ }^{2}$ Department of Mechanical and Process Engineering, ETH Zurich, 8092 Zurich, Switzerland

${ }^{3}$ Department of Chemistry and Biochemistry, University of Colorado, Boulder, Colorado 80309, United States

"email:alan.weimer@colorado.edu,charles.musgrave@,colorado.edu

*To whom correspondence should be addressed

\section{Supporting Information}

Table S1. Energies of Figure 4.

\begin{tabular}{|c|r|r|}
\hline \multicolumn{3}{|c|}{ Figure 4 Energies [kJ/mol] } \\
\hline State & Intrinsic & \multicolumn{1}{c|}{ Mediated } \\
\hline 2NH* (1) & 0 & 0 \\
\hline $\mathrm{TS}^{*}(2)$ & 331 & 89 \\
\hline $2 \mathrm{NH}^{*}(3)$ & -14 & 54 \\
\hline $\mathrm{TS}^{2}(4)$ & 398 & 198 \\
\hline $\mathrm{NH}^{*}(5)$ & 181 & 135 \\
\hline
\end{tabular}

Table S2. Energies of Figure $5 \mathbf{b}$.

\begin{tabular}{|c|r|}
\hline \multicolumn{2}{|c|}{ Figure 5b Energies [kJ/mol] } \\
\hline State & Energy \\
\hline Bare (1) & 0 \\
\hline Mol. ads. (2) & -162 \\
\hline NH$^{*}(3)$ & -193 \\
\hline 2NH* (4) & -462 \\
\hline TS1 (5) & -373 \\
\hline 2NH* (6) & -409 \\
\hline TS2 (7) & -265 \\
\hline NH2* (8) & -328 \\
\hline TS3 (9) & -133 \\
\hline NH3* (10) & -447 \\
\hline NH3 (11) & -264 \\
\hline
\end{tabular}


Table S3. Energies of Figure $6 \mathrm{e}$.

\begin{tabular}{|c|r|}
\hline \multicolumn{2}{|c|}{ Figure 6e Energies [kJ/mol] } \\
\hline State & Energy \\
\hline Bare (1) & 0 \\
\hline Mol. ads. (2) & -162 \\
\hline NH$^{*}(3)$ & -193 \\
\hline 2NH* (4) & -462 \\
\hline 3NH* (5) & -664 \\
\hline TS1 (6) & -434 \\
\hline 3NH* (7) & -447 \\
\hline TS2 (8) & -348 \\
\hline NH2* (9) & -468 \\
\hline TS3 (10) & -306 \\
\hline NH3* (11) & -385 \\
\hline TS4 (12) & -373 \\
\hline NH3* (13) & -374 \\
\hline NH3* ox. (14) & -664 \\
\hline NH3*+ox. + dis. (15) & -713 \\
\hline NH3 (16) & -523 \\
\hline
\end{tabular}

